\title{
Editorial: Journal of Trust Management
}

\author{
Stephen Marsh
}

Correspondence:

Stephen.Marsh@uoit.ca University of Ontario Institute of

Technology, Oshawa, Canada

\section{Dear reader}

Welcome! It's been a long time coming, but here at last is the first tranche of papers and the official opening of the Journal of Trust Management, a SpringerOpen publication that is dedicated to the exposition and exploration of research and development in the fields of trust management and computational trust.

This is a new journal, and still a relatively novel field, the presence of 'trust' and trust management tracks in just about all security conferences (and more than a few computer science, $\mathrm{HCI}$, etc. conferences) notwithstanding. Indeed, the presence of such tracks illustrates two things about the field - it is recognized as being important, if not vital; and it is rather often misunderstood or misappropriated. Thus, a few lines are necessary about what this journal is exploring, and what trust management and computational trust are. Bear with me, or, if you already know and don't need to read another exploration, feel free to skip to the end, where the papers in this issue are discussed, and the important people related to this journal are acknowledged.

Firstly, we should think about what this journal is and is not. It's not $100 \%$ a computer science journal. It's not $100 \%$ a social science journal. And it's not $100 \%$ a security journal. It is, however, a mixture of social, computer and security. This isn't becauase we can't define the field, it's because the field touches on, and is touched by, a great many subject areas. We therefore welcome submissions from across the board of explorations of how people use trust, particularly in situations where they are supported by computational systems. We welcome submissions related to how trust is used in security applications, from Trust-Based Access Control to Trusted Computing and all points inbetween. We equally welcome explorations of trust from the computational point of view - how systems can use it, calculate (with) it, make decisions around and with it, and justify them. How, indeed, different trust models can be used in the many places where systems make decisions, whether or not humans are in that particular loop. We also welcome examinations and expositions of research related to how humans trust technology, in fields such as Human Computer Interaction and Computer Supported Collaborative Work. In other words, we are interested in the role of trust where technology and people interact, where technology makes decisions, and where uncertainty about actions exists - and that is a lot of touch points.

The history of computational trust and trust management, though short (indeed, around 20 years), is exciting and sometimes tangled. Part of the problem is the close relationship trust has with security. Like any close relationship, it has its ups and downs and its fair share of misunderstandings, and we are just beginning to get to grips with the differences between trust and security, as well as their co-existence.

(c) 2014 Marsh; licensee Springer. This is an Open Access article distributed under the terms of the Creative Commons Attribution License (http://creativecommons.org/licenses/by/4.0), which permits unrestricted use, distribution, and reproduction in any medium, provided the original work is properly credited. 
Future observations both here, as part of a new JoTM blog, and one hopes in papers within the journal, will undoubtedly touch upon this fascinating area with much more grace than a simple editorial paragraph can do (the interested reader is however recommended to read Gollmann's expositions on the topic (Gollmann [1,2]) as a start.

The width of our interests, and the depth of the excellent research carried out there, is illustrated in the first set of articles for this journal. This is composed of a special issue set of papers selected from the best papers presented at the IFIPTM 2013 conference in Malaga, Spain and the PST 2013 conference in Tarragonia, Catalonia. I am delighted to be able to point you toward Carmen Fernandez Gago and Christian Damsgaard Jensen's editorial on that topic, and the papers included in this issue.

Before closing, indulge me whilst I take a moment to thank the people who did the real work here. Naturally, an academic journal is only as good as the reviewers and editors who make it up. We are indeed fortunate to have as Editors in Chief and on our editorial board the acknowledged leaders in the fields of computational trust and trust management, and they have put a great deal of effort and work into making this journal happen. To them I proffer my deepest gratitude for the realization of a long-held dream. To the 'behind the scenes' staff at Springer, for taking the dream and making it exist online, and more importantly as an open access journal, a great deal more thanks is due. Finally, but by no means least, Bev Ford and Rachel Roberts at Springer... I'm not at all sure how much patience Bev has, but it is both highly cherished and appreciated - and the nudges and cajolery over the past decade and a half, in various forms, has been fantastic. Rachel, probably long-suffering and certainly the best support an $\mathrm{EiC}$ can have, has shown a similar amount of patience (in less time knowing me!) and, if it's possible, and even greater ability to nudge us gently on the way to the birth of this journal. Without whom...

And without further ado, Dear Reader, enjoy our first submissions and do consider submitting your own work.

With best wishes

Stephen Marsh

Editor in Chief

Received: 8 April 2014 Accepted: 9 April 2014

Published: 20 May 2014

References

1. Gollmann D (2006) Why trust is bad for security. Electronic Notes in Theoretical Computer Science. 157(3):3-9. http://www.sciencedirect.com/science/article/pii/S1571066106002891

2. Gollmann D (2011) From access control to trust management, and back - a petition. In: Wakeman I, Gudes E, Jensen C, Crampton J (eds) Trust management V, proceedings IFIPTM. Springer IFIP AICT 358, Springer IFIP AICT, pp 1-9 